\title{
БАЗА СТАНДАРТИЗИРОВАННЫХ ИЗОБРАЖЕНИЙ ВOSS: АДАПТАЦИЯ ДЛЯ ИСПОЛЬЗОВАНИЯ НА РУССКОЯЗЫЧНОЙ ВЫБОРКЕ
}

\author{
М.С. СОПОВ ${ }^{\mathrm{a}}$ А.С. СТАРОДУБЦЕВ ${ }^{\mathrm{a}}$, К.Г. МИРОШНИК ${ }^{\mathrm{a}}$, \\ Р.Ю. ШИНДРИКОВ
}

${ }^{a}$ Санкт-Петербургский государственный университет, 199034, Россия, Санкт-Петербург, Университетская наб., д. 7/9

\section{Резюме}

Базы стандартизированных стимулов широко используются при изучении процессов зрительного восприятия и порождения речи. Они предоставляют исследователям ценную информацию о характеристиках стимульного материала, которые могут влиять на результаты экспериментов как побочные переменные. Настоящая работа посвящена русскоязычной адаптации базы стандартизированных изображений BOSS (The Bank of Standardized Stimuli) - высококачественных фотографий предметов повседневного обихода (посуды, одежды, продуктов питания и т.д.). Процедура адаптации состояла из двух этапов. В первом этапе адаптации приняли участие 45 респондентов в возрасте от 19 до 46 лет (M=24.9, $\mathrm{SD}=6.1)$. Участники исследования последовательно называли изображения, оценивали их по параметрам «знакомости» и «визуальной сложности» и относили изображения к одной из 18 категорий. Впоследствии для каждого изображения были рассчитаны следующие параметры: наиболее частотное наименование, согласованность наименований (процент респондентов, давших наиболее частотное наименование), средняя визуальная сложность, средняя знакомость, наиболее частотная категория и согласованность категорий. Фотографии, согласованность наименования которых превысила 50\% (326 изображений), использовались на втором этапе. Во втором этапе адаптации приняли участие 33 респондента в возрасте от 19 до 44 лет ( $\mathrm{M}=25.3, \mathrm{SD}=4.9)$, которые оценивали отобранные изображения по параметрам «согласованность предметов» (насколько изображение объекта согласуется с его ментальной репрезентацией), «согласованность ракурсов» и «очевидность манипуляции» (насколько легко человеку представить действие, связанное с данным предметом). Впоследствии для каждого изображения подсчитывались средние значения оцененных параметров. Результаты исследования находятся в свободном доступе и могут быть использованы при разработке различных экспериментов в области когнитивной психологии и психолингвистики.

Ключевые слова: база стандартизированных стимулов, стандартизация, адаптация, BOSS, согласованность наименований. 
Важным условием сохранения внутренней валидности экспериментального исследования является контроль побочных переменных. Для осуществления этой задачи исследователям в области экспериментальной психологии и психолингвистики необходимо иметь в распоряжении стимульный материал, перцептивные и лингвистические характеристики которого были бы заранее известны. Целью создания баз стандартизированных стимулов (БСС) являются именно выявление потенциальных побочных переменных и оценка соответствующих им параметров применительно к конкретным стимулам.

Так, одна из первых БСС, разработанная Дж. Снодграсс и М. Вандерварт в 1980 г., включает 260 черно-белых контурных рисунков предметов, каждому из которых в ходе опроса респондентов были присвоены значения по следующим параметрам: «Согласованность наименований» (процент испытуемых, давших изображенному предмету модальное наименование $\left.{ }^{1}\right)$, «Знакомость» (субъективная оценка знакомости предмета), «Визуальная сложность» (субъективная оценка сложности графического исполнения рисунка) и «Согласованность образов» (степень соответствия образа предмета, возникающего при прочтении его наименования, рисунку предмета) (Snodgrass, Vanderwart, 1980). Нормативные данные, полученные в ходе оценки перечисленных параметров, используются в экспериментальных исследованиях как для контроля побочных переменных, так и для проверки экспериментальных гипотез.

С момента публикации работы Дж. Снодграсс и М. Вандерварт было создано большое число разнообразных БСС, причем основная их часть - на материале английского языка. В многочисленных исследованиях показано, что родной язык и культурная принадлежность респондентов оказывают существенное влияние на результаты стандартизации (Bates et al., 2003; Kremin et al., 2003). Так, предметы, часто встречающиеся в определенной культуре, могут оцениваться представителями этой культуры как более знакомые и менее сложные (к примеру, круассан для французских респондентов или бейсбольная перчатка для американских респондентов) (Pompéia et al., 2003). Кроме того, языки различаются между собой по количеству синонимов, используемых для обозначения одних и тех же предметов (к примеру, в русском языке помидор часто называется томатом; в английском языке для наименования того же предмета используется только одно слово - tomato). Таким образом, область применения БСС ограничивается той культурно-языковой средой, в которой проводилась стандартизация стимулов. Это делает невозможным использование англоязычных БСС в экспериментах, проводимых на русскоязычных респондентах. Выходом из ситуации является адаптация БСС, в ходе которой респонденты из выбранной культурно-языковой среды повторно оценивают стимулы по выделенным разработчиками БСС параметрам.

\footnotetext{
${ }^{1}$ Наиболее частотное наименование предмета по результатам стандартизации стимулов. Так, револьвер из базы Дж. Снодграсс и М. Вандерварт называется большинством русскоязычных испытуемых (91\%) как «пистолет» (Григорьев и др., 2010). Данное обозначение рассматривается как модальное наименование предмета.
} 
На сегодняшний день исследователям доступны две базы стандартизированных изображений, адаптированные для работы с русскоязычной выборкой. К ним относится классическая база Дж. Снодграсс и М. Вандерварт (Григорьев и др., 2010), а также ее модификация, включающая цветные версии оригинальных рисунков (Tsaparina et al., 2011). Кроме того, на материале русского языка разработана БСС Предмет-Действие, включающая черно-белые рисунки предметов и выполняемых с ними действий (Akinina et al., 2015). Изображения из перечисленных баз позволяют решать большое число экспериментальных задач, однако их схематичность и ограниченная цветовая палитра могут негативно сказываться на экологической валидности исследований. По этой причине исследования процессов зрительного восприятия и порождения речи все чаще проводятся с использованием цветных фотографий предметов (Brodeur et al., 2010).

Целью настоящего исследования является адаптация широко используемой в экспериментальной психологии и психолингвистике базы фотографий BOSS $^{2}$ для работы с русскоязычной выборкой (Brodeur et al., 2010, 2014). Данная БСС имеет ряд преимуществ перед другими базами фотографических стимулов, такими как БСС М. Виджиано с соавт. (Viggiano et al., 2004), БСС Хатфилдский картиночный тест (The Hatfield Image Test - Adlington et al., 2009) или БСС Ф.Д. Морено-Мартинез и П.Р. Монторо (Moreno-Martinez, Montoro, 2012). Во-первых, финальная версия BOSS включает 1410 изображений, что на порядок больше, чем в других БСС ${ }^{3}$. Во-вторых, разработчики базы самостоятельно осуществляли фотосъемку предметов, а не обрабатывали фотографии, взятые из Интернета. Поскольку предметы снимались при идентичном освещении, качество фотографий из базы BOSS превышает качество фотографий из других БСС. В-третьих, разработчики базы проводили стандартизацию изображений по семи параметрам, в то время как другие фотографические стимулы стандартизированы только по четырем параметрам, выделенным Дж. Снодграсс и М. Вандерварт. К параметрам «Согласованность наименований», «Знакомость» и «Визуальная сложность» были добавлены параметры «Согласованность категорий» (процент испытуемых, причисливших предмет к модальной категории) и «Очевидность манипуляции» (субъективная оценка сложности опознания предмета по действию, обычно выполняемому с этим предметом). Кроме того, параметр «Согласованность образов» был разделен на два параметра: «Согласованность предметов» (степень соответствия образа предмета его изображению безотносительно

\footnotetext{
${ }^{2}$ Английская аббревиатура BOSS (The Bank Of Standardized Stimuli) совпадает по значению с введенной нами аббревиатурой БСС. Однако М. Бродёр с соавт. используют ее только для обозначения разработанной ими базы фотографий, в связи с чем аббревиатура BOSS также будет использоваться нами как сокращенное название.

${ }^{3}$ Стандартизация базы включала две фазы. На первой фазе респонденты оценивали 538 фотографий (Brodeur et al., 2010), на второй фазе - 930 фотографий (Brodeur et al., 2014).

${ }^{4}$ Без учета лингвистических параметров, относящихся к наименованиям изображений (таких как лексическая частотность наименований или возраст их усвоения).
} 
к ракурсу фотографии) и «Согласованность ракурсов» (аналогичный параметр, относящийся к ракурсу фотографии). Расширение перечня параметров стандартизации дает возможность исследователям контролировать большее число потенциальных побочных переменных.

Для адаптации BOSS нами были выбраны 542 изображения, большинство из которых использовались на первой фазе англоязычной стандартизации стимулов (Brodeur et al., 2010). Процедура адаптации состояла из двух этапов. На первом этапе испытуемые оценивали фотографии предметов по четырем параметрам: «Согласованность наименований», «Знакомость», «Визуальная сложность» и «Согласованность категорий». На втором этапе испытуемые из другой выборки оценивали изображения по трем параметрам: «Согласованность предметов», «Согласованность ракурсов» и «Очевидность манипуляции».

\section{Метод}

\section{Испытуемые}

В исследовании приняли участие 78 респондентов с нормальным или скорректированным до нормального зрением, свободно владеющие русским языком. Выборка исследования была разделена на две подгруппы. Первая подгруппа, состоящая из 45 респондентов в возрасте от 19 до 46 лет (19 мужчин и 26 женщин, средний возраст 24.9, SD = 6.1), была задействована на первом этапе исследования. Вторая подгруппа, состоящая из 33 респондентов в возрасте от 19 до 44 лет (14 мужчин и 19 женщин, средний возраст 25.3, SD = 4.9), была задействована на втором этапе. Респонденты не получали вознаграждения за участие в исследовании.

Поскольку для проведения исследования требовалось приблизительно три часа, каждый этап был разделен на две равные части, между которыми предполагался отдых. Обе части прошли 40 респондентов на первом этапе и 27 респондентов на втором этапе. Остальные респонденты приняли участие только в одной из частей.

Исследование было согласовано с Этическим комитетом Санкт-Петербургского государственного университета. Все респонденты приступали к выполнению задания только после подписания информированного согласия, в котором оговаривалось, что в любой момент они могут прервать исследование.

\section{Стимульный материал}

В общей сложности на первом этапе исследования использовались 542 фотографии предметов из базы изображений BOSS. Процедура создания стимулов подробно описана в статье М. Бродёра с соавт. (Brodeur et al., 2010). Стимулы, используемые при адаптации BOSS, были заимствованы нами из нескольких источников. Во-первых, 516 изображений взяты из сета стимулов, использовавшихся на первой фазе стандартизации BOSS (первый сет). 
Оригинальный сет стимулов, состоящий из 538 изображений, был уменьшен в связи с удалением 13 фотографий устаревших предметов (таких как VHS-кассета или барабан для подачи слайдов в проектор), 3 фотографий культурноспецифичных предметов (розетка северно-американского образца, канадские доллары, кепка с эмблемой НАТО) и 6 фотографий, отбракованных разработчиками базы в связи с их низким качеством. Во-вторых, 6 изображений взяты из сета стимулов, использовавшихся на второй фазе стандартизации BOSS (второй сет). Это фотографии, которыми разработчики базы заменили отбракованные стимулы из первого сета. В-третьих, 20 изображений взяты из сета стимулов, не прошедших стандартизацию (Brodeur et al., 2014). Эти фотографии включены в исследование в связи с тем, что они, с нашей точки зрения, являются более удачными изображениями соответствующих предметов из первого сета и в большей степени отвечают нуждам экспериментальных исследований. К примеру, огнетушитель на фотографии из сета нестандартизированных стимулов (fireextinguisher02.jpg) больше напоминает российские огнетушители, нежели аналогичный предмет из первого сета (fireextinguisher01.jpg).

На втором этапе исследования использовались 326 фотографий предметов, согласованность наименований которых по результатам первого этапа превысила 50\%. Стимулы с более низкими значениями согласованности наименований были удалены в связи с тем, что их модальные наименования зачастую не позволяли сгенерировать однозначные образы представления, необходимые для оценки параметров «Согласованность предметов» и «Согласованность ракурсов».

\section{Процедура}

На обоих этапах исследования испытуемым последовательно предъявлялись фотографии предметов из базы BOSS, которые необходимо было называть и оценивать по ряду параметров. Стимулы предъявлялись на дисплее компьютера, что является существенным отличием процедуры настоящего исследования от процедуры первой фазы стандартизации BOSS, в рамках которой предъявление стимулов осуществлялось при помощи проектора. Кроме того, испытуемые, участвовавшие в адаптации BOSS, не были ограничены во времени ответов, хотя в оригинальном исследовании ограничения присутствовали (на ответ давалось 5 секунд). Эти особенности соответствуют условиям проведения второй фазы стандартизации BOSS. Однако сбор ответов осуществлялся нами так же, как и на первой фазе стандартизации: испытуемые заносили ответы на специальные бумажные бланки. Инструкции также были напечатаны на бланке, находящемся перед испытуемым на протяжении всего исследования. Как было отмечено ранее, каждый из этапов исследования был разделен на две равные части. Перерывы между частями не фиксировались по времени.

На первом этапе исследования респондентам требовалось назвать предъявленный предмет, оценить его знакомость и визуальную сложность изображения, а 
также причислить предмет к одной из 18 категорий. Инструкции давались респондентам в устной форме, однако их текст дополнительно был напечатан на листке с необходимой информацией. Русскоязычные версии инструкций, использовавшиеся при адаптации BOSS, и их англоязычные аналоги из оригинального исследования представлены в таблице 1.

Названия предметов заносились респондентами на бумажный бланк. В случае если респонденту не удавалось подобрать название для предмета, ему предлагалось занести на бланк одну из следующих аббревиатур: НЗО (незнакомый объект), НКН (не знаю, как называется) или ЗКН (забыл, как называется). Параметры «Знакомость» и «Визуальная сложность» оценивались по пятибалльной шкале, где значение 1 соответствовало ответам «незнакомый предмет» либо «простой предмет», а значение 5 соответствовало ответам «знакомый предмет» либо «сложный предмет». Номер категории, к которой с точки зрения респондента относится предмет, также заносился на бланк ответов. Список категорий и соответствующих им номеров был напечатан на листке с инструкциями. Из него можно было выбрать одну из следующих категорий: строительные материалы (building materials), одежда (clothing), ювелирные изделия и подарки (decorations and gift accessories), электроника и аксессуары (electronic devices and accessories), продукты питания (food), мебель (furniture), игры и развлечения (games, toys, and entertainment), opyдия труда и аксессуары (hand labor tools and accessories), предметы домашнего обихода (household articles and cleaners), деньги и драгоценности (jewels and money), кухонная утварь (kitchen utensils), медицинские инструменты и аксессуары (medical instruments and accessories), музыкальные инструменты

Таблица 1

Инструкции, использовавшиеся на первом этапе исследования

\begin{tabular}{|l|l|l|}
\hline \multicolumn{1}{|c|}{ Параметры } & \multicolumn{1}{|c|}{ Русскоязычная инструкция } & \multicolumn{1}{c|}{$\begin{array}{c}\text { Англоязычная инструкция } \\
\text { (Brodeur et al., 2010) }\end{array}$} \\
\hline $\begin{array}{l}\text { Наименование } \\
\text { (Name) }\end{array}$ & $\begin{array}{l}\text { Постарайтесь кратко и точно } \\
\text { назвать предъявленный объект. } \\
\text { Запишите ответ в бланк ответов }\end{array}$ & $\begin{array}{l}\text { Identify the object as briefly and } \\
\text { unambiguously as possible by writ- } \\
\text { ing only one name, the first name } \\
\text { that comes to mind }\end{array}$ \\
\hline $\begin{array}{l}\text { Знакомость } \\
\text { (Familiarity) }\end{array}$ & $\begin{array}{l}\text { Оцените, насколько } \\
\text { предъявленный объект кажется } \\
\text { Вам знакомым }\end{array}$ & $\begin{array}{l}\text { Rate the level to which you are } \\
\text { familiar with the object }\end{array}$ \\
\hline $\begin{array}{l}\text { Визуальная } \\
\text { сложность (Visual } \\
\text { сотрlехіty) }\end{array}$ & $\begin{array}{l}\text { Оцените уровень визуальной } \\
\text { сложности изображения } \\
\text { (количество деталей, } \\
\text { спутанность линий) }\end{array}$ & $\begin{array}{l}\text { Subjectively rate the level to which } \\
\text { the image appears to be complex in } \\
\text { terms of the quantity of details and } \\
\text { the intricacy of the lines }\end{array}$ \\
\hline $\begin{array}{l}\text { Категория } \\
\text { (Саtegory) }\end{array}$ & $\begin{array}{l}\text { Определите, к какой из } \\
\text { нижеуказанных категорий } \\
\text { принадлежит объект }\end{array}$ & $\begin{array}{l}\text { Determine in which category the } \\
\text { object belongs to }\end{array}$ \\
\hline
\end{tabular}


(musical instruments), живая природа (natural elements and vegetation), активный отдых и спорт (outdoor activity and sport items), банные принадлежности и средства личной гигиены (skin care and bathroom items), канцелярские принадлежности и школьная утварь (stationary and school supplies), оружие и предметы, связанные с войной (weapons and items related to war). В случае если предмет не попадает ни под одну из перечисленных категорий испытуемому предлагалось занести его в категорию «другое» (others).

На втором этапе исследования респонденты оценивали стимулы по параметрам «Согласованность предметов», «Согласованность ракурсов» и «Очевидность манипуляции». Как и на первом этапе, инструкции давались в устной и письменной формах (таблица 2). Оценка параметров осуществлялась при помощи пятибалльной шкалы, на которой значение 1 соответствовало низкому уровню согласованности предметов/ракурсов или очевидности манипуляции, а значение 5 - высокому уровню.

Особенностью процедуры второго этапа является то, что перед фотографией предмета респонденту предъявлялось модальное наименование предмета. Задача респондента состояла в том, чтобы представить предмет по его модальному наименованию, а затем сличить образ представления с фотографией предмета. Близость образа и предмета требовалось оценить по двум параметрам: «Согласованность предметов» и «Согласованность ракурсов». Данные о согласованности наименований, необходимые для определения модальных наименований предметов, были собраны на первом этапе исследования.

Таблица 2

Инструкции, использовавшиеся на втором этапе исследования

\begin{tabular}{|c|c|c|}
\hline Параметры & Русскоязычная инструкция & $\begin{array}{c}\text { Англоязычная инструкция } \\
\text { (из статьи Brodeur et al., } \\
\text { 2010) }\end{array}$ \\
\hline $\begin{array}{l}\text { Согласованность } \\
\text { предметов } \\
\text { (Name agree- } \\
\text { ment) }\end{array}$ & $\begin{array}{l}\text { Оцените, насколько изображенный } \\
\text { предмет соответствует } \\
\text { представленному Вами предмету. При } \\
\text { ответе постарайтесь не учитывать угол } \\
\text { обзора (ракурс) изображения }\end{array}$ & $\begin{array}{l}\text { How closely the picture resem- } \\
\text { bles the mental image you had } \\
\text { for the object name, independ- } \\
\text { ently from its position? }\end{array}$ \\
\hline $\begin{array}{l}\text { Согласованность } \\
\text { ракурсов } \\
\text { (Viewpoint } \\
\text { agreement) }\end{array}$ & $\begin{array}{l}\text { Оцените, насколько ракурс } \\
\text { изображения соответствует } \\
\text { представленному Вами ракурсу }\end{array}$ & $\begin{array}{l}\text { How closely the object is posi- } \\
\text { tioned as the object you imag- } \\
\text { ined? }\end{array}$ \\
\hline $\begin{array}{l}\text { Очевидность } \\
\text { манипуляции } \\
\text { (Manipulability) }\end{array}$ & $\begin{array}{l}\text { Можете ли Вы без особых } \\
\text { затруднений изобразить действие, } \\
\text { связанное с предметом? Причем так, } \\
\text { чтобы другой человек мог с легкостью } \\
\text { угадать, о каком предмете идет речь? }\end{array}$ & $\begin{array}{l}\text { Could you easily mime the } \\
\text { action usually associated with } \\
\text { this object so that any person } \\
\text { looking at you doing this } \\
\text { action could decide which } \\
\text { object goes with this action? }\end{array}$ \\
\hline
\end{tabular}


Параметр «Очевидность манипуляции» оценивался в отношении фотографий предметов, а не образов представления. Для пояснения инструкции респонденты до начала исследования выполняли небольшую пробную задачу. Им необходимо было представить самолет, после чего на дисплее компьютера последовательно предъявлялись две фотографии самолета «Ту-154», снятые с разных ракурсов, и фотография самолета «Edgley EA-7 Optica». Первый из них рассматривался как типичный представитель класса «самолеты», в связи с чем предполагалось, что согласованность предметов для него будет высокой. Поскольку самолет был запечатлен с двух наиболее типичных ракурсов, предполагалось, что для одной из фотографий (ракурс которой в наибольшей степени соответствует образу представления) согласованность ракурсов будет высокой, а для другой низкой. В инструкции делался акцент на то, что параметры «Согласованность предметов» и «Согласованность ракурсов» независимы друг от друга: даже если на двух фотографиях представлен один и тот же предмет, согласованность ракурсов для них может различаться. Самолет «Edgley EA-7 Optica» демонстрировался как пример нетипичного представителя класса «самолеты», согласованность предметов для которого должна оцениваться как низкая. Респонденты переходили от модальных наименований предметов к их фотографиям посредством нажатия клавиши «Пробел». Время ответов не ограничивалось.

\section{Анализ данных}

Модальное наименование. Для каждого стимула подсчитывалось количество предложенных наименований и число испытуемых, давших эти наименования. Наиболее частотное наименование предмета (данное большинством испытуемых) обозначалось как модальное наименование. Процент испытуемых, давших предмету модальное наименование, обозначался как согласованность наименований. Ответы «НКО», «НКН» и «ЗКН» анализировались отдельно от других и не использовались при выявлении модальных наименований. В случае если два наименования были даны одинаковым числом респондентов, в качестве модального выбирался наиболее точный ответ («компьютерная мышь» является более точным наименованием, нежели «мышка»). Уменьшительно-ласкательные наименования предметов (перышко, зеркальце) рассматривались как отдельные ответы. Составные ответы, образованные двумя и более словами, подсчитывались независимо от порядка слов (ответы «стеклянная банка» и «банка стеклянная» рассматривались как идентичные). Имена прилагательные, входящие в состав наименований, исключались в случае их субъективности или иррелевантности выполняемой задаче (ответ «ваза красивая» вносился в итоговую таблицу как «ваза»).

Показатель Н (H-value). Определяется как средняя двоичная энтропия сообщения (в битах). В отличие от согласованности наименований данный показатель учитывает пропорцию респондентов, давших каждый из вариантов ответа. Он рассчитывается по формуле 


$$
H=\sum_{i=1}^{k} P_{i} \log _{2}\left(1 / P_{i}\right),
$$

где $\mathrm{H}$ - средняя двоичная энтропия сообщения, $\mathrm{k}$ - количество предложенных наименований предмета, $\mathrm{P}_{\mathrm{i}}$ - количество респондентов, давших один из вариантов наименования. Чем разнообразнее ответы испытуемых (чем больше наименований используется для обозначения предмета), тем выше будет значение показателя Н для соответствующего стимула. При расчете показателя нами не учитывались ответы «НКО», «НКН» и «ЗКН».

Модальная категория. Процедура определения модальной категории и оценки значений параметров «Согласованность категорий» и «Показатель Н для категорий» полностью соответствовала таковой для наименований предметов. Однако категория «другое» рассматривалась как полноценный ответ и могла стать модальной категорией предмета. Значения оставшихся показателей, а именно параметров «Знакомость», «Визуальная сложность», «Согласованность предметов», «Согласованность ракурсов» и «Очевидность манипуляции», вычислялись как средние оценки по пятибалльной шкале.

\section{Результаты и обсуждение}

Корреляционный анализ используемых в исследовании параметров был проведен с помощью критерия корреляции Пирсона с поправкой на множественные сравнения Бонферрони. Выявлены сходные комбинации значимо коррелирующих параметров для русскоязычной выборки и англоязычных респондентов, участвовавших в первой фазе стандартизации BOSS. Ocновные результаты исследования представлены в таблице 3. Помимо показателей описательной статистики, в ней указаны значения внутренней согласованности для параметров, оценка которых производилась при помощи пятибалльной шкалы («Знакомость», «Визуальная сложность», «Согласованность предметов», «Согласованность ракурсов» и «Очевидность манипуляции»). Значения коэффициента $\alpha$ Кронбаха для перечисленных параметров превышает 0.9, что свидетельствует о высокой степени их внутренней согласованности. Сопоставление результатов англоязычной стандартизации BOSS и ее русскоязычной адаптации приведено в таблице 4. Корреляционная матрица параметров стандартизации для двух выборок приведена в таблице 5. Со стимульным материалом и полученными в ходе адаптации данными для конкретных изображений можно ознакомиться на сайте журнала «Психология. Журнал вышей школы экономики» (https://psy-journal.hse.ru/additionalmaterials).

Несмотря на сходство результатов, полученных в ходе русскоязычного и англоязычного исследований, между корреляционными матрицами существует ряд различий, требующих пояснений. Так, нами была получена значимая корреляция между параметрами «Знакомость» и «Визуальная сложность» $(r=-0.44, p<0.001)$. Этот результат представляет интерес постольку, поскольку аналогичная корреляция наблюдается в большинстве стандартизационных исследований (см.: Brodeur et al., 2010), но не в первой фазе стандар- 
Таблица 3

Показатели описательной статистики и внутренней согласованности параметров (коэффициент $\alpha$ Кронбаха)

\begin{tabular}{|l|c|c|c|c|c|}
\hline Параметры & Среднее & $\begin{array}{c}\text { Стандартное } \\
\text { отклонение }\end{array}$ & Минимум & Максимум & $\begin{array}{c}\text { Внутренняя } \\
\text { согласованность }\end{array}$ \\
\hline $\mathrm{CH}$ & $63 \%$ & $24 \%$ & $13 \%$ & $100 \%$ & \\
\hline $\mathrm{H}_{\text {Намм }}$ & 1.59 & 0.99 & 0.00 & 4.03 & \\
\hline $\mathrm{H}$ О & $2 \%$ & $6 \%$ & $0 \%$ & $55 \%$ & \\
\hline $\mathrm{HКН}$ & $3 \%$ & $6 \%$ & $0 \%$ & $36 \%$ & \\
\hline 3 НН & $2 \%$ & $3 \%$ & $0 \%$ & $24 \%$ & \\
\hline $\mathrm{CK}$ & $75 \%$ & $22 \%$ & $22 \%$ & $100 \%$ & \\
\hline $\mathrm{H}_{\text {Кат }}$ & 1.01 & 0.73 & 0.00 & 2.73 & \\
\hline 3 & 4.19 & 0.53 & 1.49 & 4.95 & 0.92 \\
\hline $\mathrm{BC}$ & 2.11 & 0.48 & 1.17 & 3.64 & 0.93 \\
\hline $\mathrm{CП}$ & 3.49 & 0.68 & 1.80 & 4.90 & 0.93 \\
\hline $\mathrm{CP}$ & 3.35 & 0.60 & 1.73 & 4.73 & 0.91 \\
\hline $\mathrm{OM}$ & 3.12 & 0.93 & 1.29 & 4.90 & 0.96 \\
\hline
\end{tabular}

Примечание. Здесь и далее значения аббревиатур: $\mathrm{CH}$ - согласованность наименований, $\mathrm{H}_{\text {намм }}$ - показатель Н для наименований, СК - согласованность категорий, $\mathrm{H}_{\text {кат }}-$ показатель $\mathrm{H}$ для категорий, 3 - знакомость, ВС - визуальная сложность, СП - согласованность предметов, $\mathrm{CP}$ - согласованность ракурсов, ОМ - очевидность манипуляции.

Таблица 4

Значения параметров стандартизации для русскоязычной и англоязычной выборок, а также корреляции между ними

\begin{tabular}{|l|c|c|c|c|c|}
\hline \multirow{2}{*}{ Параметры } & \multicolumn{2}{|c|}{ Русскоязычные респонденты } & \multicolumn{2}{|c|}{ Англоязычные респонденты } & \multirow{2}{*}{ Корреляция } \\
\cline { 2 - 5 } & Среднее & $\begin{array}{c}\text { Стандартное } \\
\text { отклонение }\end{array}$ & Среднее & $\begin{array}{c}\text { Стандартное } \\
\text { отклонение }\end{array}$ & \\
\hline $\mathrm{CH}$ & $63 \%$ & $24 \%$ & $62 \%$ & $25 \%$ & $0.52^{*}$ \\
\hline $\mathrm{H}_{\text {Намм }}$ & 1.59 & 0.99 & 1.79 & 1.12 & $0.58^{*}$ \\
\hline $\mathrm{CK}$ & $75 \%$ & $22 \%$ & $72 \%$ & $19 \%$ & $0.64^{*}$ \\
\hline $\mathrm{H}_{\text {Кат }}$ & 1.01 & 0.73 & 1.19 & 0.68 & $0.72^{*}$ \\
\hline 3 & 4.19 & 0.53 & 3.96 & 0.47 & $0.69^{*}$ \\
\hline $\mathrm{BC}$ & 2.11 & 0.48 & 2.39 & 0.43 & $0.84^{*}$ \\
\hline $\mathrm{CП}$ & 3.49 & 0.68 & 3.88 & 0.54 & $0.65^{*}$ \\
\hline $\mathrm{CP}$ & 3.35 & 0.60 & 3.71 & 0.49 & $0.69^{*}$ \\
\hline $\mathrm{OM}$ & 3.12 & 0.93 & 2.54 & 0.79 & $0.89^{*}$ \\
\hline
\end{tabular}

Примечание. ${ }^{*} p<0.05$ (с учетом поправки Бонферрони). Значения параметров для англоязычной выборки могут отличаться от представленных в статье М. Бродёра и коллег (Brodeur et al., 2010), поскольку последние рассчитывались после исключения части изображений. 
Таблица 5

Структура корреляций параметров (в скобках приведены значения корреляции, полученные на англоязычной выборке)

\begin{tabular}{|c|c|c|c|c|c|c|c|c|}
\hline & CH & $\mathbf{H}_{\text {Наим }}$ & СК & $\mathbf{H}_{\text {Кат }}$ & 3 & BC & CII & CP \\
\hline $\mathrm{H}_{\text {Наим }}$ & $\begin{array}{c}-0.95^{*} \\
\left(-0.94^{*}\right)\end{array}$ & & & & & & & \\
\hline СК & $\begin{array}{l}0.16^{*} \\
(0.14)\end{array}$ & $\begin{array}{c}-0.20^{*} \\
\left(-0.14^{*}\right)\end{array}$ & & & & & & \\
\hline $\mathrm{H}_{\text {Кат }}$ & $\begin{array}{c}-0.17^{*} \\
\left(-0.19^{*}\right)\end{array}$ & $\begin{array}{c}0.21^{*} \\
\left(0.20^{*}\right)\end{array}$ & $\begin{array}{c}-0.97^{*} \\
\left(-0.95^{*}\right)\end{array}$ & & & & & \\
\hline 3 & $\begin{array}{c}0.46^{*} \\
\left(0.51^{*}\right)\end{array}$ & $\begin{array}{c}-0.48^{*} \\
\left(-0.53^{*}\right)\end{array}$ & $\begin{array}{c}0.38^{*} \\
\left(0.33^{*}\right)\end{array}$ & $\begin{array}{c}-0.40^{*} \\
\left(-0.40^{*}\right)\end{array}$ & & & & \\
\hline $\mathrm{BC}$ & $\begin{array}{c}-0.13 \\
(-0.07)\end{array}$ & $\begin{array}{c}0.13 \\
(0.08)\end{array}$ & $\begin{array}{l}-0.20^{*} \\
(-0.05)\end{array}$ & $\begin{array}{l}0.19^{*} \\
(0.05)\end{array}$ & $\begin{array}{l}-0.44^{*} \\
(-0.13)\end{array}$ & & & \\
\hline СП & $\begin{array}{c}0.32^{*} \\
\left(0.37^{*}\right)\end{array}$ & $\begin{array}{c}-0.37^{*} \\
\left(-0.38^{*}\right)\end{array}$ & $\begin{array}{c}0.21^{*} \\
\left(0.21^{*}\right)\end{array}$ & $\begin{array}{c}-0.23^{*} \\
\left(-0.26^{*}\right)\end{array}$ & $\begin{array}{c}0.46^{*} \\
\left(0.42^{*}\right)\end{array}$ & $\begin{array}{l}-0.23^{*} \\
(-0.09)\end{array}$ & & \\
\hline $\mathrm{CP}$ & $\begin{array}{c}0.08 \\
\left(0.21^{*}\right)\end{array}$ & $\begin{array}{c}-0.08 \\
\left(-0.2^{*}\right)\end{array}$ & $\begin{array}{c}0.11 \\
\left(0.16^{*}\right)\end{array}$ & $\begin{array}{c}-0.09 \\
\left(-0.19^{*}\right)\end{array}$ & $\begin{array}{c}0.05 \\
\left(0.23^{*}\right)\end{array}$ & $\begin{array}{c}-0.09 \\
(-0.02)\end{array}$ & $\begin{array}{c}0.34^{*} \\
\left(0.59^{*}\right)\end{array}$ & \\
\hline $\mathrm{OM}$ & $\begin{array}{c}0.17 \\
\left(0.24^{*}\right)\end{array}$ & $\begin{array}{c}-0.16 \\
\left(-0.26^{*}\right)\end{array}$ & $\begin{array}{l}-0.18^{*} \\
(-0.03)\end{array}$ & $\begin{array}{l}0.18^{*} \\
(0.05)\end{array}$ & $\begin{array}{c}0.11 \\
\left(0.21^{*}\right)\end{array}$ & $\begin{array}{c}0.03 \\
(0.04)\end{array}$ & $\begin{array}{c}0.01 \\
(0.10)\end{array}$ & $\begin{array}{c}-0.11 \\
(-0.03)\end{array}$ \\
\hline
\end{tabular}

Примечание. ${ }^{*} p<0.05$ (с учетом поправки Бонферрони). Значения параметров для англоязычной выборки могут отличаться от представленных в статье М. Бродёра и коллег (Brodeur et al., 2010), поскольку последние рассчитывались после исключения части изображений.

тизации BOSS $(r=-0.13, p>0.1)$. Разработчики базы связывают отсутствие корреляции между параметрами с тем, что все фотографии в BOSS являются изображениями высокочастотных предметов, применяемых в каждодневном обиходе. Данная особенность резко отличает BOSS от существующих баз контурных рисунков, включающих изображения редких, но легко опознаваемых предметов (как, например, изображения экзотических животных). Наличие подобных стимулов дает возможность использовать более однозначный критерий определения знакомости предметов, а именно объективную встречаемость предметов в жизни респондентов. При оценке же знакомости бытовых предметов приходится пользоваться более интуитивными критериями. Разработчики базы не поясняют, почему способ оценки знакомости стимулов должен определять наличие корреляции между параметрами «Знакомость» и «Визуальная сложность», однако можно допустить, что низкочастотные предметы требуют более подробной прорисовки для того, чтобы быть легко опознаваемыми, нежели предметы каждодневного обихода. При оценке же визуальной сложности испытуемые в первую очередь ориентируются на детализацию изображений (данный момент оговаривается в инструкции; см. таблицу 1). 
Таким образом, визуальная сложность рисунков коррелирует с объективной встречаемостью предметов, а последняя используется респондентами для оценки знакомости.

Данная гипотеза подтверждается результатами второй фазы стандартизации BOSS, в которой, помимо фотографий бытовых предметов, использовались также фотографии животных, растений, транспортных средств и архитектурных форм. В ней была обнаружена значимая корреляция между параметрами «Знакомость» и «Визуальная сложность». Однако та же корреляция была обнаружена и в настоящем исследовании, включающем стимулы из первой фазы стандартизации BOSS. Данный результат расходится с предположением разработчиков базы. Он может быть объяснен либо культурно-языковыми особенностями русскоязычной выборки, либо возрастом участников адаптации. Второе объяснение кажется нам более обоснованным. Так, в оригинальном исследовании средний возраст респондентов, оценивавших степень знакомства предметов и визуальную сложность фотографий, составлял 33.6 года $(\mathrm{SD}=12.7)$, а в настоящем исследовании - 24.9 года $(\mathrm{SD}=6.1)$. Причем демографические данные участников адаптации BOSS в большей степени соответствуют данным респондентов, участвовавших в стандартизациях контурных рисунков (Brodeur et al., 2010). Серьезным подтверждением нашей гипотезы является возраст участников второй фазы стандартизации BOSS, оценивавших параметры «Знакомость» и «Визуальная сложность». Он составляет 25.2 года (SD = 7.5) (Brodeur et al., 2014).

Возраст испытуемых может влиять на корреляцию показателей «Знакомость» и «Визуальная сложность» из-за того, что молодые респонденты используют более интуитивную стратегию оценки знакомости предметов. Они в большей мере опираются на «чувство знакомости», нежели взрослые респонденты, ориентирующиеся в первую очередь на объективную частотность предметов. Согласно теории Л. Якоби и К. Уайтхаус, интенсивность чувства знакомости (feel of familiarity) зависит от беглости переработки информации: стимул воспринимается как более знакомый в случае, если на его переработку тратится меньшее количество ресурсов (Jacoby, Whitehouse, 1989; Lanska et al., 2014). В свою очередь, беглость переработки информации зависит не только от опыта взаимодействия со стимулом, но и от его перцептивной сложности. Таким образом, визуально простые изображения воспринимаются как более знакомые. Испытуемые, использующие интуитивную стратегию оценки знакомости, становятся жертвами данной иллюзии.

Другой особенностью полученных нами результатов является отсутствие корреляции между параметром «Согласованность ракурсов» и всеми остальными параметрами за исключением согласованности предметов. В англоязычной стандартизации BOSS этот показатель коррелирует со всеми параметрами, кроме визуальной сложности. Данный результат вызывает сложности при его интерпретации. На сегодняшний день оценка параметра «Согласованность ракурсов» осуществлялась только в рамках двух фаз стандартизации BOSS и настоящего исследования; этих данных недостаточно для того, чтобы сделать вывод о причинах обсуждаемого различия. То же справедливо и в 
отношении корреляции факторов «Знакомость» и «Очевидность манипуляции», обнаруженной в оригинальном исследовании, но отсутствующей в полученных нами данных. Для прояснения этих результатов требуются дополнительные исследования с использованием стимулов из базы изображений BOSS.

\section{Заключение}

В исследовании представлены результаты адаптации стандартизированной базы изображений BOSS для русскоязычной выборки. Результаты исследования свидетельствуют о сходстве комбинаций значимо коррелирующих параметров для англоязычной и русскоязычной выборок. Фотографии из адаптированной базы BOSS могут быть использованы в широком спектре экспериментальных исследований для систематического контроля побочных переменных, связанных с особенностями стимульного материала.

\section{Литература}

Григорьев, А. А., Ощепков, И. В., Балясникова, О. В., Орлова, Е. А. (2010). Нормативные данные по образности, согласованности наименований, знакомости и соответствию наименования изображению для набора из 286 стимулов. Вестник Московского государственного областного университета. Серия: Психологические науки, 1, 42-51.

Ссылки на зарубежные источники см. в разделе References после англоязычного блока.

Сопов Михаил Сергеевич - аспирант, Санкт-Петербургский государственный университет. Сфера научных интересов: селективное внимание, неосознанные психические процессы, опознание стимулов, лексический доступ, нейрональные механизмы памяти, аналитическая философия сознания.

Контакты: mikhail.sopov@gmail.com

Стародубцев Алексей Сергеевич - аспирант, Санкт-Петербургский государственный университет.

Сфера научных интересов: когнитивная психология, психология памяти, устойчивые ошибки, явления интерференции, научение, обработка противоречивой информации, когнитивный контроль.

Контакты: fleksbr@yandex.ru

Мирошник Кирилл Геннадьевич - студент, Санкт-Петербургский государственный университет.

Сфера научных интересов: когнитивная психология, психология творчества.

Контакты: cyril.miroshnik@gmail.com

Шиндриков Роман Юрьевич - аспирант, Санкт-Петербургский государственный университет. Сфера научных интересов: селективное внимание, когнитивный контроль, нейропсихологическая диагностика.

Контакты: shindrikov@list.ru 


\title{
The Bank of Standardized Stimuli (BOSS): Adaptation for Use in Russian-Language Studies
}

\author{
M.S. Sopova, A.S. Starodubtsev ${ }^{\mathrm{a}}$, K.G. Miroshnik ${ }^{\mathrm{a}}$, R.Yu. Shindrikov ${ }^{\mathrm{a}}$ \\ ${ }^{a}$ Saint Petersburg State University, 7/9 Universitetskaya emb., Saint Petersburg, 199034, Russian Federation
}

\begin{abstract}
Standardized sets of stimuli are widely applied in studies on visual perception and speech production. They provide valuable information about a range of parameters that can be controlled as confounding variables in experiments. The present study provides Russian-language normative data for 542 pictures from the Bank of Standardized Stimuli (BOSS), which contains high-quality photos of everyday objects (such as kitchen utensils, clothes, food, etc.). The study design consisted of two stages. In the first stage, 45 respondents aged from 19 to $46(\mathrm{M}=24.9, \mathrm{SD}=6.1)$ were asked to name given objects, rate them on "familiarity" and "visual complexity", and select in which of 18 categories they belong to. Afterward, the following parameters were determined for each image: modal name, modal name agreement (percentage of respondents who chose the most frequently used name for an object), mean visual complexity, mean familiarity, modal category, and category agreement. Photos with the modal name agreement greater than 50\% (326 items) were taken for the second stage. In the second stage, 33 respondents aged from 19 to $44(\mathrm{M}=25.3, \mathrm{SD}=4.9)$ rated selected pictures on "object agreement" (the degree to which photo of an object corresponds to its mental image), "viewpoint agreement", and "manipulability" (the degree to which it is easy to imagine an action that is highly associated with this specific object). Eventually, mean values for all rated parameters were computed. The results are freely available online and can be used for conducting various experiments in the field of cognitive psychology and psycholinguistics.
\end{abstract}

Keywords: bank of standardized stimuli, standardization, adaptation, BOSS, name agreement.

\section{References}

Adlington, R. L., Laws, K. R., \& Gale, T. M. (2009). The Hatfield Image Test (HIT): A new picture test and norms for experimental and clinical use. Journal of Clinical and Experimental Neuropsychology, 31(6), 731-753. doi:10.1080/13803390802488103

Akinina, Y., Malyutina, S., Ivanova, M., Iskra, E., Mannova, E., \& Dragoy, O. (2015). Russian normative data for 375 action pictures and verbs. Behavior Research Methods, 47, 691-707. doi:10.3758/s13428-014-0492-9

Bates, E., D’Amico, S., Jacobsen, T., Szŭkely, A., Andonova, E., Devescovi, A., ... Wicha, N. (2003). Timed picture naming in seven languages. Psychonomic Bulletin and Review, 10(2), 344-380. doi:10.3758/BF03196494

Brodeur, M. B., Dionne-Dostie, E., Montreuil, T., \& Lepage, M. (2010). The Bank of Standardized Stimuli (BOSS), a new set of 480 normative photos of objects to be used as visual stimuli in cognitive research. PLoS ONE, 5(5), e10773. doi:10.1371/journal.pone.0010773

Brodeur, M. B., Guérard, K., \& Bouras, M. (2014). Bank of standardized stimuli (BOSS) phase II: 930 new normative photos. PLoS ONE, 9(9), e106953. doi:10.1371/journal.pone.0106953 
Grigoryev, A., Oshchepkov, I., Balyasnikova, O., \& Orlova, E. (2010). Normative data shape, coherence names, friends, and that match images for a set of incentives 286. Vestnik Moskovskogo Gosudarstvennogo Oblastnogo Universiteta. Seriya: Psychologicheskie Nauki, 1, 42-51. (in Russian)

Jacoby, L. L., \& Whitehouse, K. (1989). An illusion of memory: False recognition influenced by unconscious perception. Journal of Experimental Psychology: General, 118(2), 126-135. doi:10.1037/0096-3445.118.2.126

Kremin, H., Akhutina, T., Basso, A., Davidoff, J., De Wilde, M., Kitzing, P., ... Weniger, D. (2003). A cross-linguistic data bank for oral picture naming in Dutch, English, German, French, Italian, Russian, Spanish, and Swedish (PEDOI). Brain and Cognition, 53(2), 243-246. doi:10.1016/S0278-2626(03)00119-2

Lanska, M., Olds, J. M., \& Westerman, D. L. (2014). Fluency effects in recognition memory: Are perceptual fluency and conceptual fluency interchangeable? Journal of Experimental Psychology: Learning, Memory, and Cognition, 40(1), 1-11. doi:10.1037/a0034309

Moreno-Martinez, F. J., \& Montoro, P. R. (2012). An ecological alternative to Snodgrass \& Vanderwart: 360 high quality colour images with norms for seven psycholinguistic variables. PLoS ONE, 7(5), e37527. doi:10.1371/journal.pone.0037527

Pompéia, S., Miranda, M. C., \& Bueno, O. F. A. (2003). Brazilian standardised norms for a set of pictures are comparable with those obtained internationally. Arquivos de Neuro-Psiquiatria, 61(4), 916-919. doi:10.1590/S0004-282X2003000600005

Snodgrass, J. G., \& Vanderwart, M. (1980). Standardized set of 260 pictures: Norms for name agreement, image agreement, familiarity, and visual complexity. Journal of Experimental Psychology: Human Learning and Memory, 6(2), 174-215. doi:10.1037/0278-7393.6.2.174

Tsaparina, D., Bonin, P., \& Méot, A. (2011). Russian norms for name agreement, image agreement for the colorized version of the Snodgrass and Vanderwart pictures and age of acquisition, conceptual familiarity, and imageability scores for modal object names. Behavior Research Methods, 43, 10851099. doi:10.3758/s13428-011-0121-9

Viggiano, M. P., Vannucci, M., \& Righi, S. (2004). A new standardized set of ecological pictures for experimental and clinical research on visual object processing. Cortex, 40(3), 491-509. doi:10.1016/S0010-9452(08)70142-4

Mikhail S. Sopov - Ph.D. student, Saint Petersburg State University. Research area: selective attention, non-conscious mental processes, identification of stimuli, lexical access, neuronal mechanisms of memory, analytic philosophy of consciousness.

E-mail: mikhail.sopov@gmail.com

Alexei S. Starodubtcev - Ph.D. student, Saint Petersburg State University.

Research area: cognitive psychology, psychology of memory, persistent errors, interference phenomena, learning, conflicting information processing, cognitive control.

E-mail: fleksbr@yandex.ru

Kirill G. Miroshnik - student, Saint Petersburg State University.

Research area: cognitive psychology, psychology of creativity.

E-mail: cyril.miroshnik@gmail.com

Roman Yu. Shindrikov - Ph.D. student, Saint Petersburg State University.

Research area: selective attention, cognitive control, neuropsychological diagnostics.

E-mail: shindrikov@list.ru 